\title{
Percutaneous pulmonary valve implantation as an alternative to repeat open-heart surgery for patients with pulmonary outflow obstruction: a reality in Singapore
}

\author{
Lik Wui Edgar Tay ${ }^{1}$, MBBs, Wei Luen James Yip ${ }^{1}$, MBBS, Ting Ting Low ${ }^{1}$, MBBS, Chin Ling William Yip ${ }^{2,3}$, MD, FRCPE, \\ Kok Fai William Kong ${ }^{1}$, MD, FRCP, Tiong Cheng $\underline{\text { Yeo }}^{1}$, MBBS, Huay Cheem $\underline{\operatorname{Tan}}^{1}$, MBBS, FRCP, \\ Shakeel Ahmed Quereshi ${ }^{4}, *$ MBChB, FRCP, Swee Chye $\underline{\text { uek }}^{2}, *, \mathrm{MD}, \mathrm{FRCPCH}$
}

Right ventricle to pulmonary artery (RV-PA) conduits have been used for the surgical repair of congenital heart defects. These conduits frequently become stenosed or develop insufficiency with time, necessitating reoperations. Percutanous pulmonary valve implantation (PPVI) can delay the need for repeated surgeries in patients with congenital heart defects and degenerated RV-PA conduits. We presented our first experience with PPVI and described in detail the procedural methods and the considerations that are needed for this intervention to be successful. Immediate and short-term clinical outcomes of our patients were reported. Good haemodynamic results were obtained, both angiographically and on echocardiography. PPVI provides an excellent alternative to repeat open-heart surgery for patients with congenital heart defects and degenerated RV-PA conduits. This represents a paradigm shift in the management of congenital heart disease, which is traditionally managed by open-heart surgery.

Keywords: percutaneous pulmonary valve implantation, pulmonary outflow obstruction

\section{INTRODUCTION}

Percutaneous pulmonary valve implantation (PPVI) was first conceptualised by Bonhoeffer et al in 2000,(1) providing an alternative treatment option to open surgical replacement for patients with congenital heart disease and degenerated right ventricle to pulmonary artery (RV-PA) conduits. Valved conduits consist of synthetic material or homograft/xenograft tissue. Their disadvantages are the inability to keep pace with the growth of the patient, mechanical distortion, and progressive degeneration leading to conduit stenosis over time. Regurgitation may also occur as the valve leaflets degenerate. These patients often require multiple sternotomies throughout their lifespan because the surgical conduits have limited durability, necessitating ongoing intermittent replacement. Each redo cardiac surgery is associated with higher technical operative difficulty and becomes increasingly hazardous, with greater mortality and morbidity risks. $^{(2,3)}$ PPVI has been shown to extend the duration of these conduits, thus reducing the total burden of redo surgeries for these patients.

For this study, we selected our patients with close attention to the indications and contraindications for this procedure, listed in Box 1.(4) A majority of our patients had Tetralogy of Fallot or pulmonary atresia/stenosis, or needed further intervention following complex congenital heart surgery, such as the Ross procedure or the Rastelli procedure. The Ross procedure is a form of cardiac surgery in which a diseased aortic valve is replaced by the patient's own pulmonary valve. A pulmonary allograft (taken from a cadaver) is then used to replace the patient's pulmonary valve. A Rastelli procedure involves using an aortic or pulmonary homograft to relieve right ventricular outlet obstruction. Some studies suggest that $50 \%$ of these homografts require replacement after about ten years. ${ }^{(5)}$

In the case of RV-PA conduit obstruction, the efficacy of PPVI on clinical outcome is well-established, ${ }^{(6,7)}$ and the European Society of Cardiology guidelines ${ }^{(4)}$ are precise in recommending the timing for early intervention. On the other hand, pulmonary regurgitation has been well tolerated for many years and the impact of early PPVI in these patients is more controversial. ${ }^{(5,7)}$ It has been suggested that the degree of right ventricular dilation should prompt the PPVI indication, rather than the severity of the pulmonary regurgitation. ${ }^{(8)}$

The Melody ${ }^{\mathrm{TM}}$ transcatheter pulmonary valve (Medtronic Inc, Minneapolis, MN, USA), the first commercially available transcatheter valve in the world, received European certification and Health Canada approval in 2006. It is a bioprosthetic valve harvested from a bovine jugular vein and sutured within a balloon-expandable platinum-iridium stent. The valve size is $18 \mathrm{~mm}$ in diameter, which is crimped to $6 \mathrm{~mm}$ and can then be balloon-expanded to three sizes $-18 \mathrm{~mm}, 20 \mathrm{~mm}$ and $22 \mathrm{~mm}$ - depending on the balloon on which it is mounted. The Melody valve is the most commonly used valve in the world in the pulmonary position, and most of the available data for PPVI is from its use. ${ }^{(9)}$

In this case series, we present our first experience with PPVI using the Melody valve for patients with RV-PA conduit stenosis in Singapore. The procedural methods and the considerations

${ }^{1}$ Department of Cardiology, National University Heart Centre Singapore, ${ }^{2}$ Department of Pediatrics, National University of Singapore, ${ }^{3}$ Department of Paediatric Cardiology, Gleneagles Hospital, Singapore, ${ }^{4}$ Department of Paediatric Cardiology, Evelina London Children's Hospital, London, UK

*These two authors contributed equally as senior author in this work.

Correspondence: DrEdgar Tay, Department of Cardiology, National University Hospital, NUHS Tower Block Level 9, 1E Kent Ridge Road, Singapore 119228. edgar_tay@nuhs.edu.sg 
Box 1. Selection criteria for percutaneous pulmonary valve implantation among patients with right ventricle to pulmonary artery conduit obstruction:

Indication

- Asymptomatic patients with severe right ventricular outflow tract (RVOT) and/or severe pulmonary regurgitation should be considered when at least one of the following criteria is present:

- Decrease in exercise capacity (cardiopulmonary exercise testing)

- Progressive right ventricular dilation $\left(>150 \mathrm{~mL} / \mathrm{m}^{2}\right)$

- Progressive right ventricular systolic dysfunction

- Progressive tricuspid regurgitation (at least moderate)

- Right ventricular systolic pressure $>80 \mathrm{mmHg}$ (tricuspid regurgitation velocity $>7.3 \mathrm{~m} / \mathrm{s}$ )

- Sustained atrial/ventricular arrhythmias

\section{Contraindication}

- Occluded central veins

- Active infection

- Unfavourable morphology of RVOT or conduit ( $<16 \mathrm{~mm}$ or $>22 \mathrm{~mm}$ )

- High-risk coronary anatomy

- Body weight $<20 \mathrm{~kg}$ (issues with cannula size)

needed for a successful intervention are described in detail. The immediate and short-term clinical outcomes of our patients for whom PPVI was performed are also reported.

\section{METHODS}

The Melody valve was implanted through a dedicated delivery system (Ensemble ${ }^{\mathrm{TM}}$ Transcatheter Delivery System, Medtronic Inc, MN, USA) consisting of a balloon-in-balloon system that allows repositioning during the valve delivery (Fig. 1). The outer balloon has three sizes (18 mm, $20 \mathrm{~mm}$ or $22 \mathrm{~mm}$ ). The valve is manually crimped onto the balloon and a retractable sheath is used to cover the balloon and the valve. The entire system has a profile (outer diameter) of 22 French ( $\mathrm{Fr}$ ) and is delivered via the femoral vein without any additional sheaths.

Careful consideration was essential to select the appropriate patients from the cohort of adult congenital heart patients followed at our institution. Investigations such as transthoracic echocardiography, cardiac magnetic resonance imaging, computed tomography (CT) angiography, cardiopulmonary exercise test and right heart catheterisation were performed for preprocedural patient screening and assessment. A dedicated heart team comprising paediatric cardiologists, adult cardiologists, imaging specialists and congenital cardiothoracic surgeons made the joint decisions on eligibility for PPVI and procedural planning.

Femoral venous access was obtained percutaneously and haemodynamic assessment was performed to assess conduit stenosis and severity of RV-PA gradient and/or pulmonary regurgitation. Angiography of the right ventricular outflow tract (RVOT) and conduit was performed for various outflow tract measurements. A 14-Fr 75-cm long Mullins sheath (Cook
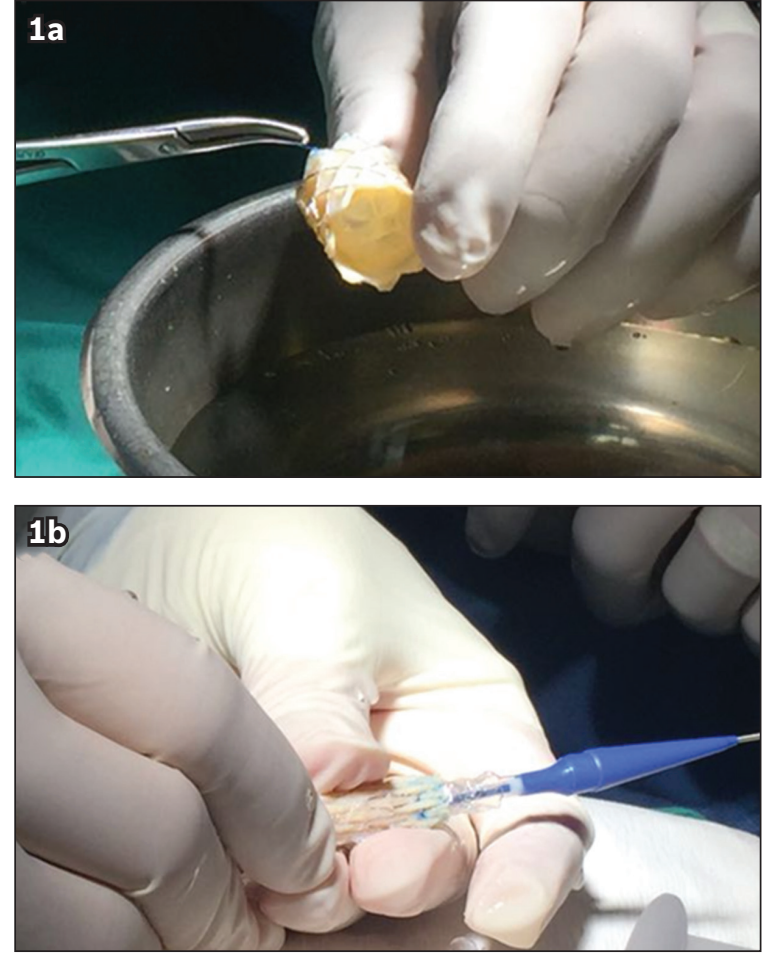

Fig. 1 Photographs show the (a) Melody valve and (b) Ensemble Transcatheter Delivery System.

Medical, Bloomington, IN, USA) was placed with its tip just distal to the conduit over a 260-cm long stiff guide wire, such as a Lunderquist wire (Cook Medical). A noncompliant balloon was then inflated within the landing zone of the RV-PA conduit to recheck measurements, and simultaneous left coronary angiography was performed to assess the risk of coronary artery compression. This test step was essential, as inflating the balloon during stent or valve implantation may cause extrinsic compression of the coronary artery if it is in close proximity. After this, the conduit was pre-stented with bare or covered stents, depending on whether calcification was present. This was an improved procedural modification over the initial cases of Melody valve implantation. The additional step of pre-stenting the conduit conveyed radial strength and reduced tension on the Melody valve, thereby reducing the risk of fracture of the valve stent. Importantly, as conduits develop significant calcification over the years, covered stents act as a safety harness in case of conduit rupture or dissection during dilation. After pre-stenting the conduit, any residual narrowing can be addressed by repeated balloon dilatation using a higher pressure noncompliant balloon. Thereafter, the Melody valve was implanted within this pre-stented scaffold. Invasive haemodynamic assessments were then performed using a multi-track catheter (NuMED Inc, Hopkinton, NY, USA) and final angiography was performed to assess the results.

\section{CASE SERIES \\ Patient 1}

A 31-year-old man with Tetralogy of Fallot had undergone initial surgical repair at four years of age and RV-PA conduit insertion 

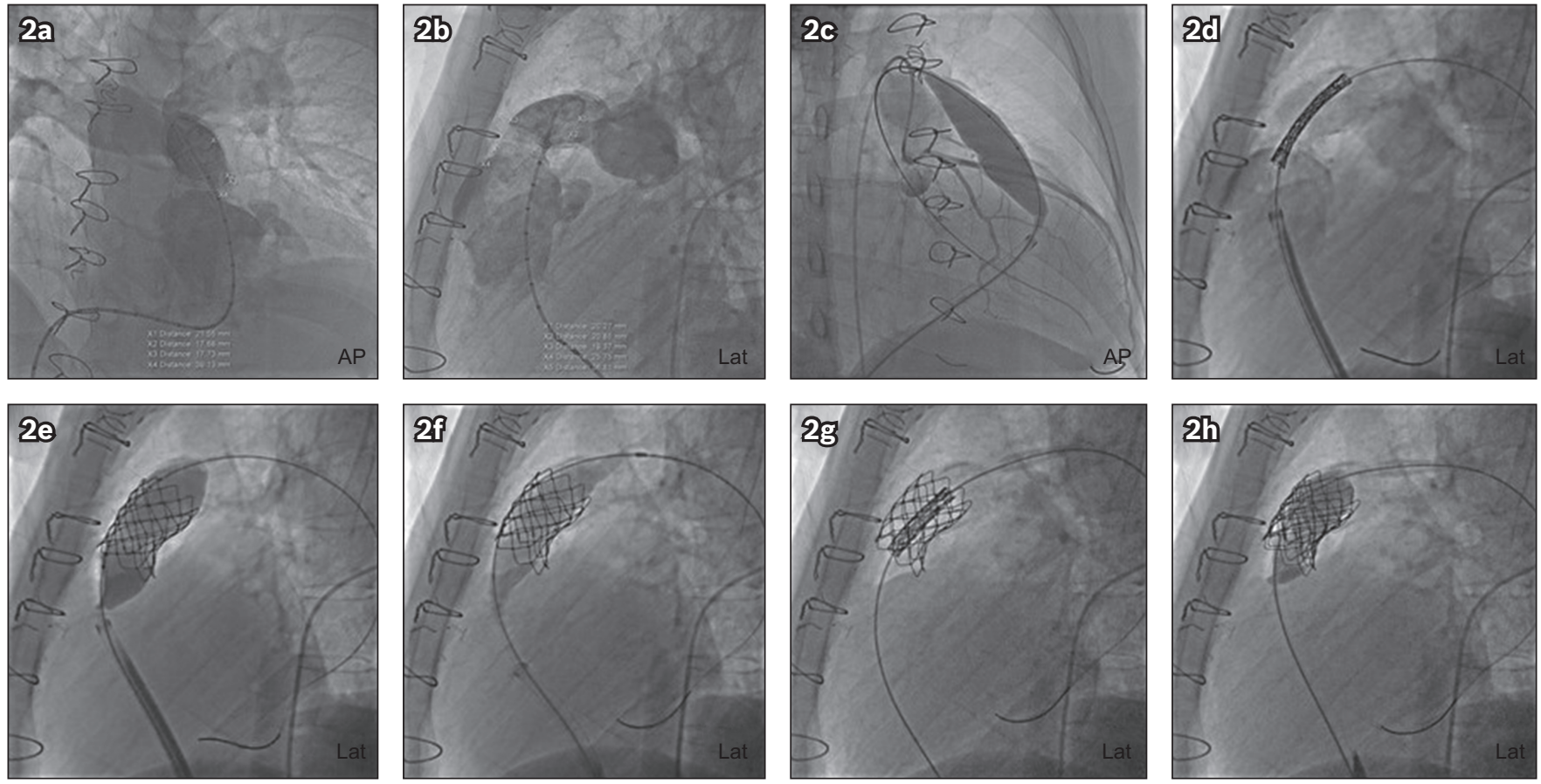

Fig. 2 Patient 1: Angiographic projections in the ( $\mathrm{a} \& \mathrm{c}$ ) anteroposterior and ( $b$ \& $d-h$ ) lateral views during a procedure show (a \& b) measurements of the conduit; (c) balloon inflation for assessment of the conduit and coronary artery interaction; pre-stenting, with (d) placement and (e) deployment of the covered stent; (f) post-dilatation of the covered stent with the Atlas balloon; and implantation, with (g) placement and (h) deployment of the Melody valve. AP: anteroposterior; Lat: lateral

using a $21-\mathrm{mm}$ pulmonary homograft 13 years later. This was followed seven years later by repeat open-heart surgery for homograft re-replacement using an aortic homograft of $20 \mathrm{~mm}$. The third open-heart surgery was complicated by severe bleeding from adhesions and a stormy recovery requiring prolonged stay in the intensive care unit. Subsequently, the patient was found to have progressive elevation of the RVOT pressure gradients from $25 \mathrm{mmHg}$ to $44 \mathrm{mmHg}$ over five years. He became symptomatic with complaints of lethargy and palpitations, requiring treatment with oral amiodarone for episodes of supraventricular tachycardia. Cardiac magnetic resonance imaging performed six months prior to PPVI showed moderate homograft stenosis and mild pulmonary regurgitation. The mean right ventricular end-diastolic volume was $160 \mathrm{~mL} / \mathrm{m}^{2}$. Right ventricular ejection fraction was reduced to $41 \%$. The most recent transthoracic echocardiography (three weeks prior to $\mathrm{PPVI}$ ) showed an estimated right ventricular systolic pressure of $78 \mathrm{mmHg}$, with severe right ventricular dysfunction and moderate tricuspid regurgitation. His cardiopulmonary exercise test showed a reduced peak $\mathrm{VO}_{2}$ max of $18 \mathrm{~mL} / \mathrm{kg} /$ minute (33\% of predicted). With these criteria, the patient fulfilled the indications for PPVI.

The procedure was performed under general anaesthesia. Two ProGlide sutures were used to preclose the femoral venous access, which was sequentially dilated to permit the placement of a 14-Fr 75-cm long Mullins sheath. Pretreatment peak-topeak gradient was $25 \mathrm{mmHg}$ under general anaesthesia and in the presence of reduced right ventricular function. Initial angiographic measurements were performed to assess the conduit size as well as to ensure no compromise of the coronary artery (Figs. 2a-c). Pre-stenting was performed with placement of a premounted $24 \mathrm{~mm} \times 45 \mathrm{~mm}$ covered Cheatham-Platinum $(\mathrm{CP})$ stent (Figs. $2 \mathrm{~d} \&$ e). Further post-dilatation of the covered stent was performed with a $20 \mathrm{~mm} \times 20 \mathrm{~mm}$ Atlas balloon (Bard, Covington, GA, USA) to $10 \mathrm{~atm}$ initially and then to $12 \mathrm{~atm}$ (Fig. 2f). A 22-mm Melody valve was subsequently implanted (Figs. $2 \mathrm{~g} \& \mathrm{~h}$ ). Postimplantation assessment showed that the peak-to-peak gradient had reduced to $5 \mathrm{mmHg}$, with no pulmonary regurgitation. Haemostasis was achieved with the ProGlide sutures for closure.

The patient was treated with aspirin 100 mg once daily and clopidogrel $75 \mathrm{mg}$ once daily for one month and subsequently planned for lifelong aspirin. He was also given intravenous cefazolin perioperatively and five days of oral augmentin. Although transthoracic echocardiography two days after PPVI showed a similar RVOT peak gradient of $40 \mathrm{mmHg}$ (no change from preprocedure echocardiography), right ventricular systolic dysfunction had improved remarkably from severe to mild. The preprocedure RV-PA gradient had probably been underestimated due to severe right ventricular systolic dysfunction. There was no pericardial effusion, and the leaflets were functioning well without pulmonary regurgitation. The patient was discharged well on the fifth postoperative day.

\section{Patient 2}

A 21-year-old man with truncus arteriosus Type 1 not associated with DiGeorge syndrome underwent a Rastelli operation with closure of the ventricular and atrial septal defects and division of patent ductus arteriosus at 29 days of age. A redo cardiac surgery was performed two years later, involving a 17-mm homograft revision and right pulmonary artery angioplasty (Fig. 3a). This surgery was complicated by severe bleeding, renal dysfunction, 

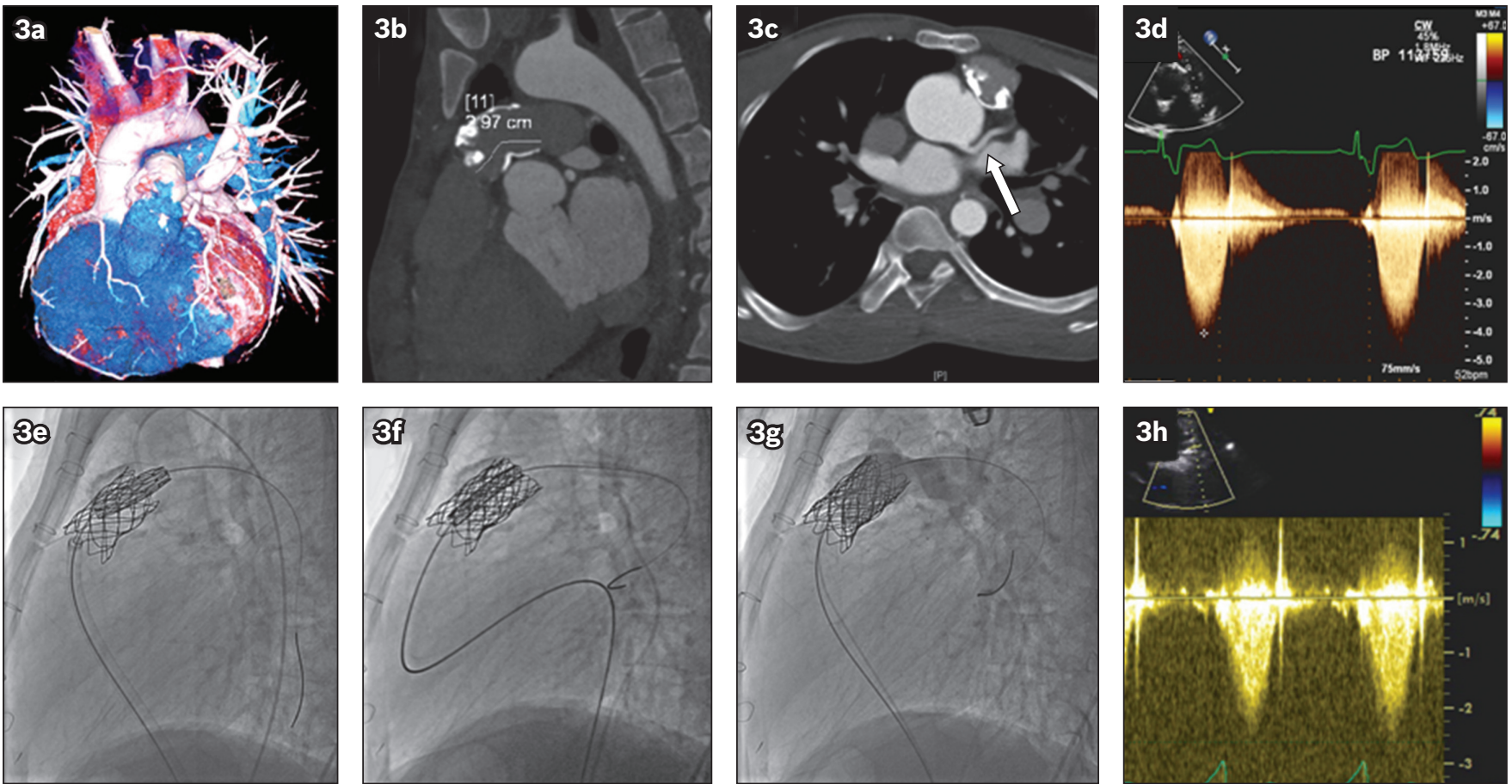

Fig. 3 Patient 2: CT images show (a) anatomy of the conduit post-Rastelli repair for truncus arteriosus; (b) the calcified conduit (length 2.97 cm); and (c) relationship between the left coronary artery (arrow) and the conduit. (d) Transthoracic echocardiogram shows high right ventricular outflow tract (RVOT) pressure gradients before Melody valve implantation. Fluoroscopy images show (e) pre-stenting, with placement of a second covered stent; and implantation, with (f) placement and (g) deployment of the Melody valve, while transthoracic echocardiogram shows (h) improvement in RVOT pressure gradients after Melody valve implantation.

gastrointestinal bleeding, pneumonia and left vocal cord palsy. During the subsequent follow-up, serial imaging showed progressive dilatation of the right ventricle to $179 \mathrm{~mL} / \mathrm{m}^{2}$. The initial moderate stenosis and regurgitation of the conduit (on cardiac magnetic resonance imaging a year previously) had progressed to severe stenosis and regurgitation on follow-up echocardiography. Estimated right ventricular systolic pressure had increased to $80 \mathrm{mmHg}$, with severe tricuspid valve regurgitation. Although the patient did not report symptoms, functional impairment was demonstrated on cardiopulmonary exercise testing, with a peak $\mathrm{VO}_{2}$ max of $14 \mathrm{~mL} / \mathrm{kg} /$ minute $(48 \%$ of predicted).

During pre-procedural planning, extensive calcification of the conduit was noted on cardiac CT (Fig. 3b). This is indicative that prior stenting of the conduit is required before pulmonary valve implantation. Care should be taken to avoid compression of the left coronary artery during pre-stenting (Fig. 3c), as they are closely related. Pre-procedural echocardiogram showed a high RVOT peak gradient of $65 \mathrm{mmHg}$ (Fig. 3d). Pre-stenting was achieved using a $20 \mathrm{~mm} \times 45 \mathrm{~mm}$ covered CP stent deployed at $4 \mathrm{~atm}$, followed by another $20 \mathrm{~mm} \times 34 \mathrm{~mm}$ covered CP stent deployed at $4 \mathrm{~atm}$ and placed more distally but overlapping with the first (Fig. 3e). This was because fracture of the calcification in the conduit had been noted posteriorly following the implantation of the first stent, but without frank extravasation of contrast. A 22-mm Melody valve was then implanted (Figs. $3 f \& g$ ) and the patient recovered uneventfully.

Transthoracic echocardiography performed the next day after PPVI showed an improvement of $40 \mathrm{mmHg}$ in RVOT peak gradient, to $25 \mathrm{mmHg}$ (Fig. 3h). The moderate right ventricular dysfunction remained unchanged; improvements in right ventricular function may only become more apparent on repeat imaging after a few weeks. There was no pericardial effusion and the leaflets were functioning well without pulmonary regurgitation. The patient was treated with oral augmentin for five days. Repeat CT pulmonary angiography performed after 14 days showed improvement. He was discharged well on the fifth postoperative day.

\section{DISCUSSION}

To the best of our knowledge, this is the first experience of transcatheter PPVI for patients with congenital heart defects and degenerated RV-PA conduits in Singapore.

Given the overall improved life expectancy of patients with surgically corrected congenital heart defects over the decades, ${ }^{(10)}$ the role of interventional therapy such as PPVI should be expected to expand, as this cohort continues to survive longer and grow in number. The short-term safety profile of the procedure is well established. The mid-term data has also been reassuring, with the recent publication of an up-to-seven-year follow-up of the Melody valve that had a median follow-up time of 4.5 years. ${ }^{(11)}$ Risks for such procedures, however, persist and include: (a) conduit rupture; (b) coronary artery compression/occlusion; (c) infective endocarditis; and (d) valve stent fracture.

Partial or total conduit rupture may follow balloon predilation and are especially likely to occur in very stenotic and highly calcified conduits. The risk of conduit rupture requiring rescue surgery can be up to $2 \% .{ }^{(12,13)}$ We used covered stents in our patients to mitigate against this risk, so that any extravasation due to conduit rupture may be confined. Most of the time, this complication of conduit rupture may be managed in the catheterisation laboratory. ${ }^{(14,15)}$ 
Coronary artery compression or occlusion can be fatal, as it may lead to acute myocardial infarction and occurs in about $1 \%$ of Melody valve implantations. ${ }^{(12,13)}$ Evaluation with prior CT coronary angiography and balloon testing during the procedure is useful for identifying high-risk cases, allowing timely preventive measures to be taken. The incidence of coronary artery compression during balloon testing ranges from $4.7 \%$ to $6 \%$. $^{(16)}$

There has been some recent concern over the rate of infective endocarditis reported post PPVI. ${ }^{(12,13)}$ To date, the estimated annualised rate of a first episode of infective endocarditis is $2.4 \%$ per patient-year. ${ }^{(17)}$ This could be related to slow flow and residual turbulence in the conduits. There could also be occult infection in the previous homograft/conduit for which the Melody valve was implanted. Infection rates of the Contegra conduit were reported to be as high as $11.3 \%$ (12 out of 106 patients) in those implanted, at a median follow-up of 4.4 years. ${ }^{(18)}$ It is important to emphasise that for these patients, antibiotic prophylaxis and good dental hygiene are of utmost importance to reduce the risk of subsequent endocarditis. ${ }^{(19)}$

It has been reported that valve stent fracture can be mitigated by modifying the procedure, adopting systematic pre-stenting of the conduit. ${ }^{(20)}$ This complication occurred at a rate of about $20 \%$ in earlier series, ${ }^{(21)}$ which has been reduced to $5 \%-16 \%$ after pre-stenting. ${ }^{(12,13)}$ Melody valve stent fractures are generally well tolerated but can potentially lead to the loss of structural integrity, embolisation or restenosis. ${ }^{(22)}$

After the PPVI procedure, it is recommended to start patients on dual antiplatelet therapy for one month and subsequent lifelong aspirin. In the immediate follow-up period, the patient should also be advised to report any new-onset arrhythmias or worsening of pre-existing arrhythmia. The incidence of postimplant arrhythmias was reported to be $15 \%$ in a recent study, but a majority of these arrhythmias were resolved by six months on follow-up. ${ }^{(23)}$ Finally, it is imperative that patients are educated on the need for antibiotic prophylaxis and encouraged to be meticulous in maintaining good dental hygiene.

The longevity of surgical RV-PA conduits is known, but longterm data with PPVI is relatively scarce. Around $50 \%$ of RV-PA conduits require replacement within ten years. ${ }^{(5)}$ Subsequent conduits may have shorter survival than the original. ${ }^{(24)}$ PPVI allows concomitant relief of RVOT obstruction and regurgitation while maintaining pulmonary valve competence. ${ }^{(14)}$ At five years after PPVI, 76\% of patients remain free from reintervention and $92 \%$ of patients remain free from explant. ${ }^{(11)}$

The risks described above and the longevity of the valve implant have to be taken into careful consideration during patient selection, weighing them against the increased operative risks associated with redo open-heart surgery. Generally, the overall lower procedure-related morbidity, together with other advantages such as a shorter hospital stay, less pain and faster recovery, make PPVI an attractive alternative to repeated sternotomies.

In conclusion, PPVI provides an excellent alternative to open-heart surgery in patients with congenital heart disease and degenerated RV-PA conduits. The importance of heart team collaboration, detailed preprocedural assessment and postprocedural care, especially for the prevention of infective endocarditis post PPVI, cannot be overemphasised.

\section{REFERENCES}

1. Bonhoeffer P, Boudjemline $Y$, Saliba Z, et al. Percutaneous replacement of pulmonary valve in a right-ventricle to pulmonary-artery prosthetic conduit with valve dysfunction. Lancet 2000; 356:1403-5.

2. Elahi MM, Dhannapuneni R, Firmin R, Hickey M. Direct complications of repeat median sternotomy in adults. Asian Cardiovasc Thorac Ann 2005; 13:135-8.

3. Elahi MM, Kirke R, Lee D, Dhannapuneni RR, Hickey MS. The complications of repeat median sternotomy in paediatrics: six-months of follow-up of consecutive cases. Interact Cardiovasc Thorac Surg 2005; 4:356-9. Retraction in: Interact Cardiovasc Thorac Surg 2014; 19:886.

4. Baumgartner $\mathrm{H}$, Bonhoeffer $\mathrm{P}$, De Groot NM, et al; Task Force on the Management of Grown-up Congenital Heart Disease of the European Society of Cardiology (ESC); Association for European Paediatric Cardiology (AEPC); ESC Committee for Practice Guidelines (CPG). ESC Guidelines for the management of grown-up congenital heart disease (new version 2010). Eur Heart J 2010; 31:2915-57.

5. Ong K, Boone R, Gao M, et al. Right ventricle to pulmonary artery conduit reoperations in patients with tetralogy of fallot or pulmonary atresia associated with ventricular septal defect. Am J Cardiol 2013; 111:1638-43.

6. Lurz P, Giardini A, Taylor AM, et al. Effect of altering pathologic right ventricular loading conditions by percutaneous pulmonary valve implantation on exercise capacity. Am J Cardiol 2010; 105:721-6.

7. Lurz P, Nordmeyer J, Giardini A, et al. Early versus late functional outcome after successful percutaneous pulmonary valve implantation: are the acute effects of altered right ventricular loading all we can expect? J Am Coll Cardiol 2011; 57:724-31

8. Vezmar M, Chaturvedi R, Lee KJ, et al. Percutaneous pulmonary valve implantation in the young 2-year follow-up. JACC Cardiovasc Interv 2010; 3:439-48.

9. Hascoët $S, A c a r P$, Boudjemline $Y$. Transcatheter pulmonary valvulation: current indications and available devices. Arch Cardiovasc Dis 2014; 107:625-34.

10. Warnes CA. The adult with congenital heart disease: born to be bad? J Am Coll Cardiol 2005; 46:1-8.

11. Cheatham JP, Hellenbrand WE, Zahn EM, et al. Clinical and hemodynamic outcomes up to 7 years after transcatheter pulmonary valve replacement in the US melody valve investigational device exemption trial. Circulation 2015; 131:1960-70.

12. Eicken A, Schubert S, Hager A, et al. Percutaneous tricuspid valve implantation: two-center experience with midterm results. Circ Cardiovasc Interv 2015; 8:e002155.

13. McElhinney DB, Hellenbrand WE, Zahn EM, et al. Short- and medium-term outcomes after transcatheter pulmonary valve placement in the expanded multicenter US melody valve trial. Circulation 2010; 122:507-16.

14. Lurz P, Coats L, Khambadkone $S$, et al. Percutaneous pulmonary valve implantation: impact of evolving technology and learning curve on clinical outcome. Circulation 2008; 117:1964-72.

15. Khambadkone S. Percutaneous pulmonary valve implantation. Ann Pediatr Cardiol 2012; 5:53-60.

16. Fraisse A, Assaidi A, Mauri L, et al. Coronary artery compression during intention to treat right ventricle outflow with percutaneous pulmonary valve implantation: incidence, diagnosis, and outcome. Catheter Cardiovasc Interv 2014; 83:E260-8.

17. McElhinney DB, Benson LN, Eicken A, et al. Infective endocarditis after transcatheter pulmonary valve replacement using the Melody valve: combined results of 3 prospective North American and European studies. Circ Cardiovasc Interv 2013; 6:292-300.

18. Albanesi F, Sekarski N, Lambrou D, Von Segesser LK, Berdajs DA. Incidence and risk factors for Contegra graft infection following right ventricular outflow tract reconstruction: long-term results. Eur J Cardiothorac Surg 2014; 45:1070-4.

19. Chen SJ, Liu CJ, Chao TF, et al. Dental scaling and risk reduction in infective endocarditis: a nationwide population-based case-control study. Can J Cardiol 2013; 29:429-33.

20. Nordmeyer J, Lurz P, Khambadkone S, et al. Pre-stenting with a bare metal stent before percutaneous pulmonary valve implantation: acute and 1-year outcomes. Heart 2011; 97:118-23.

21. McElhinney DB, Cheatham JP, Jones TK, et al. Stent fracture, valve dysfunction, and right ventricular outflow tract reintervention after transcatheter pulmonary valve implantation: patient-related and procedural risk factors in the US Melody Valve Trial. Circ Cardiovasc Interv 2011; 4:602-14.

22. Butera G, Milanesi O, Spadoni I, et al. Melody transcatheter pulmonary valve implantation. Results from the registry of the Italian Society of Pediatric Cardiology. Catheter Cardiovasc Interv 2013; 81:310-6.

23. Nguyen HH, Shahanavaz S, Van Hare GF, et al. Percutaneous pulmonary valve implantation alters electrophysiologic substrate. J Am Heart Assoc 2016; 5:e004325.

24. Stark JC, Bull C, Stajevic M, et al. Fate of subpulmonary homograft conduits: determinants of late homograft failure. J Thorac Cardiovasc Surg 1998; 115:506-16. 